\title{
Evaluation of Preoperative Carcinoembryonic Antigen Positivity in Relation to Volume-based PET/CT Parameters in Patients with Colorectal Cancer
}

\author{
(1) Yunus Güzel, (1) Cihan Gündoğan, (1) Canan Can
}

University of Health Sciences Turkey, Diyarbakır Gazi Yaşargil Training and Research Hospital, Clinic of Nuclear Medicine, Diyarbakır, Turkey

\section{Abstract}

Objective: This study aimed to determine the relationship between the tumor load and carcinoembryonic antigen (CEA) positivity by comparing CEA positivity and volume-based positron emission tomography/computed tomography (PET/CT) preoperative parameters in patients with colorectal cancer.

Methods: This retrospective study included a total of 87 patients with colorectal cancer who underwent PET/CT between January 2018 and December 2019 and had simultaneous CEA measurements. CEA level $\geq 5 \mathrm{ng} / \mathrm{mL}$ was accepted as positive. Patients who underwent surgery or received chemotherapy/radiotherapy were excluded from the study. The maximum standardized uptake value (SUV ${ }_{\text {max }}$ ), metabolic tumor volume (MTV), and total lesion glycolysis (TLG) values were calculated using ${ }^{18}$ fluoro-fluorodeoxyglucose PET/CT images of the patients.

Results: CEA was positive in $43(49.4 \%)$ patients. The site of the primary lesion was the rectum in 40 (46\%) patients, sigmoid colon in 19 (21.8\%), ascending colon in 14 (16.1\%), descending colon in 9 (10.3\%), transverse colon in $3(3.4 \%)$, hepatic flexure in 1 (1.1\%), and descendingsigmoid colon junction in 1 (1.1\%). No significant association was noted between the intestinal segment of the primary lesion and CEA positivity $(\mathrm{p}=0.878)$. Moreover, no significant difference was found among SUV ${ }_{\max }$, MTV, and TLG values of the primary tumor and CEA positivity $(p=0.611, p=0.980$, and $p=0.527$, respectively).

Conclusion: Preoperatively, no significant relationship was found between CEA positivity and volume-based PET/CT parameters, specifically MTV and TLG, in patients with colon cancer. CEA positivity in the preoperative period has low diagnostic effectiveness independent of the tumor load.

Keywords: FDG PET/CT, colorectal carcinoma, carcinoembryonic antigen, metabolic tumor volume, total lesion glycolysis

\section{INTRODUCTION}

Colorectal cancer is the third leading cancer type and one of the most common causes of cancer-related deaths (1). Colorectal cancers are diagnosed by colonoscopic and histopathological examination, and tumor markers are important for screening and detecting recurrences. Carcinoembryonic antigen (CEA) is one of the main tumor markers used in diagnosing colorectal cancers $(2,3)$. CEA has a glycoprotein structure, and preoperative and postoperative measurements of its serum level have been considered clinically useful (4).
${ }^{18}$ fluoro-fluorodeoxyglucose $\quad\left({ }^{18} \mathrm{~F}-\mathrm{FDG}\right)$ positron emission tomography/computed tomography (PET/CT) is a frequently used imaging method for metabolic characterization, staging, treatment response evaluation, radiotherapy planning, and restaging of colorectal cancers (5). The maximum standardized uptake value $\left(\mathrm{SUV}_{\max }\right)$ measured with ${ }^{18} \mathrm{~F}-\mathrm{FDG} \mathrm{PET} / \mathrm{CT}$ is a preoperative prognostic factor for patients with colorectal cancer (6). Recently, some volume-based metabolic parameters such as the metabolic active tumor volume (MTV) and total lesion glycolysis (TLG) have also been studied as prognostic factors $(7,8)$. 
To our knowledge, only a limited number of studies have investigated the relationship between serum CEA levels and volume-based PET/CT parameters in patients with colorectal cancer, and most of these studies focused on recurrence assessment $(9,10)$. Thus, in the present study, we aimed to determine the relationship between the tumor load and CEA positivity by comparing preoperative period CEA positivity and volume-based PET/CT parameters in patients with colorectal cancer.

\section{METHODS}

This retrospective study included 87 patients with colorectal cancer who underwent PET/CT and had simultaneous CEA measurements between January 2018 and December 2019 at the department of nuclear medicine in Gazi Yasargil Training and Research Hospital. Overall, 23 patients were female and 64 were male, and their mean age was $56.7 \pm 15.44$ years. Patients with an interval of more than 1 week between CEA measurement and ${ }^{18} \mathrm{~F}$-FDG PET/CT and patients with distant organ and lymph node metastases found in PET/CT were excluded from the study. Patients with M0 and N0 status were included in the study. Participant selection was performed during the preoperative period. Furthermore, patients who received chemotherapy/ radiotherapy were excluded. All patients who met the inclusion criteria were consecutively included in the study. SUV ${ }_{\max }, \mathrm{MTV}$, and TLG values were calculated by using the ${ }^{18} \mathrm{~F}$-FDG PET/CT images of these patients. Collected data and CEA results were compared statistically.

This study was conducted in accordance with the local good clinical practice guidelines and current legislations. Permission was obtained from the Institutional Ethics Committee of the University Health Science Turkey of Gazi Yasargil Training and Research Hospital for the use and publication of patient data (protocol no: 435/2020).

\section{${ }^{18}$ F-FDG PET/CT Imaging Protocol}

All patients were instructed not to eat at least $6 \mathrm{~h}$ before the intervention, and intravenous administration of glucose was stopped. Before the injection of ${ }^{18} \mathrm{~F}-\mathrm{FDG}$, blood glucose values were confirmed to be $\leq 140 \mathrm{mg} / \mathrm{dL}$ by finger stick measurement method. Moreover, 60 min after the injection of 3.5-5.5 MBq/kg ${ }^{18} \mathrm{~F}-\mathrm{FDG}$, CT was performed from the supine position using the Discovery IQ 4 ring $20 \mathrm{~cm}$ axial field of view (FOV) PET/CT scanner (GE Healthcare, Milwaukee, WI, USA) in the supine position, from the vertex to the middle of the thigh. The scanning parameters were as follows: Tube voltage, $120 \mathrm{kV}$; tube current, $80 \mathrm{mAs} / \mathrm{slice}$; FOV, $700 \mathrm{~mm}$, transaxial without gap; collimation, $64 \times 0.625$ $\mathrm{mm}$; pitch, 1.4; rotation time, $0.5 \mathrm{~s}$; slice thickness, $3.3 \mathrm{~mm}$; matrix size, $512 \times 512$. Then, 2.5 min bedside PET images were obtained at three-dimensional FOV of $20 \mathrm{~cm}$, ordered subset expectation-maximization algorithm of 5 iterations/12 subsets, and full width at half maximum of $3 \mathrm{~mm}$.

\section{Analysis of Images}

All ${ }^{18} \mathrm{~F}$-FDG PET/CT images were evaluated using PET volume computerized assisted reporting software (GE Advantage Workstation software version AW 4.7, USA) by two nuclear medicine specialists with at least 10 years of experience. The volumetric region of interest was drawn manually from the primary lesion in the colon or rectum in three planes, and the lesion and automatic MTV, TLG (MTV $\times$ SUV $\left._{\text {mean }}\right)$, and SUV ${ }_{\text {max }}$ values were obtained by the device for each lesion using $40 \%$ SUV threshold (Figure 1).

\section{Statistical Analysis}

Statistical analysis was performed using IBM SPSS Statistics for Windows version 25.0 (IBM Corp., Armonk, NY). The normality of continuous variables was evaluated by the Shapiro-Wilk-Francia test, and variance homogeneity was evaluated by Levene test. For the comparison of two independent groups in terms of quantitative data, independent-samples t-test was used together with Bootstrap results, while the Mann-Whitney $U$ test was used with Monte Carlo simulation technique. Fisher's exact test results were used to compare categorical data, while the FisherFreeman-Halton test was used with Monte Carlo simulation results. The correlation analysis of the variables was performed by Spearman's rho test. Quantitative variables are expressed as

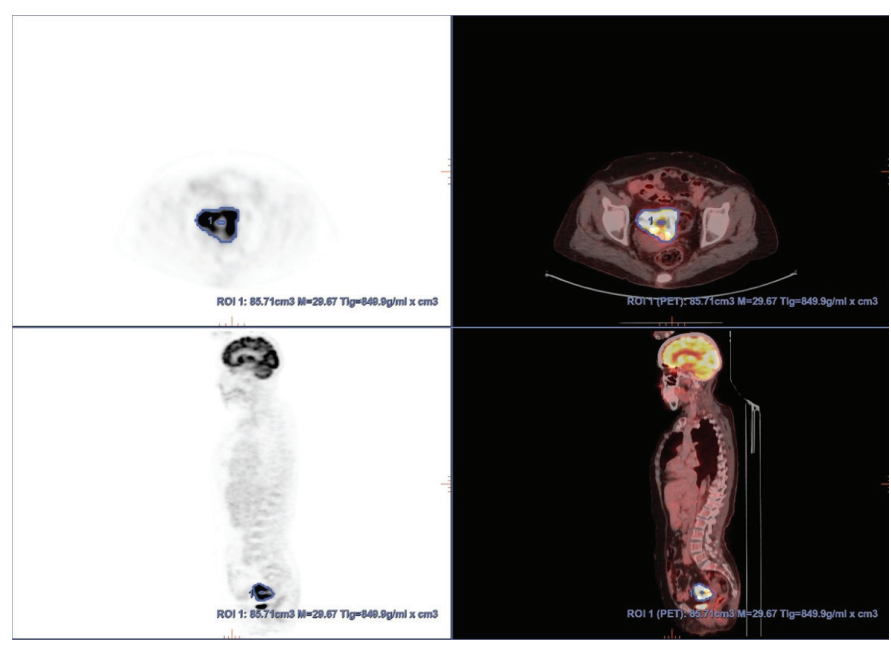

Figure 1. A 39-year-old female patient with sigmoid colon adenocarcinoma. Primary tumor metabolic tumor volume, $85.71 \mathrm{~cm}^{3}$; primary tumor total lesion glycolysis, $849.9 \mathrm{~g} / \mathrm{mL} \times \mathrm{cm}^{3}$; primary tumor SUV $_{\max }$, 29.67; carcinoembryonic antigen, $47.4 \mathrm{ng} / \mathrm{mL}$

SUV $_{\max }$ : Maximum standardized uptake value 
mean \pm standard deviation and median (minimum-maximum), and categorical variables are shown as $n$ (\%). Variables were examined at a 95\% confidence level, and $p<0.05$ was considered significant.

\section{RESULTS}

CEA was positive in 43 (49.4\%) patients (Table 1). No significant difference was noted in CEA positivity with respect to age and gender ( $p=0.150, p=0.811$, respectively). The site of the primary lesion was distributed as follows: Rectum, 40 (46\%) cases; sigmoid colon, 19 (21.8\%) cases; ascending colon, 14 (16.1\%) cases; descending colon, 9 (10.3\%) cases; transverse colon, 3 (3.4\%) cases; hepatic flexure, 1(1.1\%) case; and descending-sigmoid colon junction, 1 (1.1\%) case (Table 1).
No significant association was noted between the intestinal segment involving the primary lesion and CEA positivity $(p=0.878)$. No significant difference was also noted among SUV $_{\max }$, MTV, and TLG values of the primary tumor and CEA positivity ( $p=0.611, p=0.980, p=0.527$, respectively) (Table 1$)$.

No significant correlation was found in Spearman's correlation test between CEA levels and PET parameters ( $p>0.05)$ (Table 2).

\section{DISCUSSION}

Few studies have compared serum levels of CEA and volumebased PET/CT parameters in patients with colorectal cancer, and most of these have focused on evaluation of recurrence $(9,10)$. Thus, in the present study, we aimed to determine the

\begin{tabular}{|c|c|c|c|c|}
\hline & \multicolumn{3}{|l|}{ CEA } & \multirow{4}{*}{ p } \\
\hline & \multirow{2}{*}{$\begin{array}{l}\text { Total } \\
(n=87)\end{array}$} & \multirow{2}{*}{$(n=44)$} & \multirow{2}{*}{$+(n=43)$} & \\
\hline & & & & \\
\hline & Mean \pm SD & Mean \pm SD & Mean \pm SD & \\
\hline \multirow[t]{2}{*}{ Age } & $56.7 \pm 15.44$ & $54.3 \pm 14.78$ & $59.1 \pm 15.91$ & $0.150^{t}$ \\
\hline & $\mathrm{n}(\%)$ & $\mathrm{n}(\%)$ & n (\%) & \\
\hline \multicolumn{5}{|l|}{ Gender } \\
\hline Female & $23(26.4)$ & $11(25.0)$ & $12(27.9)$ & $0.811^{\mathrm{fe}}$ \\
\hline Male & 64 (73.6) & $33(75.0)$ & $31(72.1)$ & - \\
\hline \multicolumn{5}{|l|}{ Lesion location } \\
\hline Ascending colon & $14(16.1)$ & $8(18.2)$ & $6(14.0)$ & $0.878^{\mathrm{ff}}$ \\
\hline Hepatic flexure & $1(1.1)$ & $1(2.3)$ & $0(0.0)$ & - \\
\hline Descending colon & $9(10.3)$ & $4(9.1)$ & $5(11.6)$ & - \\
\hline Descending colon-sigmoid & $1(1.1)$ & $0(0.0)$ & $1(2.3)$ & - \\
\hline Rectum & $40(46.0)$ & $21(47.7)$ & $19(44.2)$ & - \\
\hline Sigmoid colon & $19(21.8)$ & $8(18.2)$ & $11(25.6)$ & - \\
\hline \multirow[t]{2}{*}{ Transverse colon } & $3(3.4)$ & $2(4.5)$ & $1(2.3)$ & - \\
\hline & Median (min-max) & Median (min-max) & Median (min-max) & \\
\hline Primary TM MTV & $34.8(3.2-256)$ & $32.935(3.2-183)$ & $34.8(3.43-256)$ & $0.980^{\mathrm{u}}$ \\
\hline Primary TM TLG & $263.7(12.3-3164)$ & 240.65 (13.1-2642) & $332.9(12.3-3164)$ & $0.527^{u}$ \\
\hline Primary TM SUV $_{\max }$ & $17.7(6.3-62.8)$ & $16.82(6.7-62.8)$ & $18.4(6.3-42.2)$ & $0.611^{\mathrm{u}}$ \\
\hline \multicolumn{5}{|c|}{ 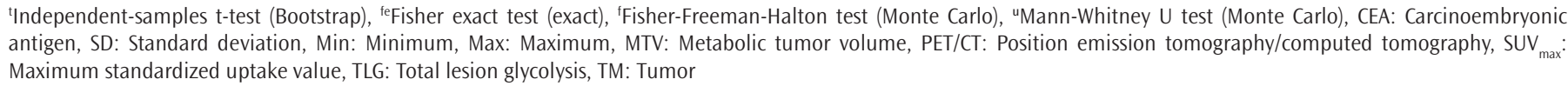 } \\
\hline
\end{tabular}

Table 2. Correlation between CEA level and metabolic and volume-based PET/CT parameters

\begin{tabular}{|l|l|l|l|l|l|l|}
\hline & \multicolumn{2}{|l|}{ Primary tumor MTV } & \multicolumn{2}{l|}{ Primary tumor TLG } & \multicolumn{2}{l|}{ Primary tumor SUV } \\
\hline \multirow{3}{*}{ CEA U/mL } & $\mathrm{r}$ & $\mathrm{p}$ & $\mathrm{r}$ & $\mathrm{p}$ & $\mathrm{r}$ & $\mathrm{p}$ \\
\cline { 2 - 6 } & 0.034 & 0.758 & 0.119 & 0.272 & 0.149 & 0.168 \\
\hline
\end{tabular}


relationship between tumor load and CEA positivity by comparing CEA positivity and volume-based PET/CT parameters of patients with colorectal cancer in the preoperative period.

In the present study, CEA positivity was not associated with age and gender. Moreover, the sensitivity of serum CEA level in the diagnosis of colorectal cancer was $49.4 \%$. In a previous study, Dbouk et al. (11) reported that CEA had diagnostic sensitivity of $58.3 \%$, which was relatively higher than our findings. This difference was considered due to the difference in the cutoff values for CEA positivity used in our study $(5 \mathrm{ng} / \mathrm{mL})$ and in their study $(3.8 \mathrm{ng} / \mathrm{mL})$ and the fact that a higher sensitivity is expected with the use of lower cutoff values (11).

In the present study, $46 \%$ of the tumoral lesions were detected in the rectum, $33.2 \%$ in the descending-sigmoid colon junction, $3.4 \%$ in the transverse colon, $1.1 \%$ in the hepatic flexure, and $16.1 \%$ in the ascending colon. Similarly, Siregar and Sibarani (12) evaluated tumor localization in colorectal cancer and reported that lesions were predominantly located in the rectum and leftsided colon.

Moreover, we observed no significant correlation between CEA positivity and the intestinal segment of the primary lesion. Siregar and Sibarani (12) reported that the highest CEA level was found in the rectum among the segments of the large intestine. Although their results appear different from our findings, the data compared are not exactly the same.

${ }^{18} \mathrm{~F}-\mathrm{FDG} \mathrm{PET} / \mathrm{CT}$ is now considered a powerful tool for the evaluation and follow-up of patients with cancer. In a retrospective study, Shi et al. (6) examined 107 patients and found that the SUV ${ }_{\max }$ value and TNM classification obtained in PET/CT were independent predictors of survival, while SUV $_{\text {max }}$ values $\leq 11.85$ were associated with better survival. However, another study showed that a high SUV $_{\text {max }}$ value in patients with resectable colorectal cancer was not significantly related with tumor recurrence and diseasefree survival (13). The tumor markers CEA and CA 19-9 were also considered to show low diagnostic performance, while high CA 19-9 levels along with PET/CT use were considered to indicate poor prognosis (14). Vallam et al. (15) reported that PET/CT is a significant tool in the detection of recurrent disease during follow-up, independent of serum CEA levels, and the probability of disease recurrence is directly proportional to the value of the increased CEA level. Another study reported the recurrence rates of $10 \%, 45 \%, 70 \%, 94 \%$, and $100 \%$ for serum CEA levels of $<5$, $5.1-10,10.1-15,15.01-50$, and $>50 \mathrm{ng} / \mathrm{mL}$, respectively (16). In addition, PET/CT scan performed in all of these patients revealed sensitivity, specificity, positive predictive, and negative predictive values of $92.7 \%, 95.2 \%, 96.2 \%$, and $90.9 \%$, respectively (15).
The consensus report by the College of American Pathologists in 1999 accepted that the tumor volume did not have a prognostic significance (category 4), but serum CEA levels gained prognostic significance (category 1) (16).

Limited studies have shown the correlation between volumebased PET/CT parameters and tumor markers. In a study of 489 patients, Kim et al. (17) did not find a correlation between pretreatment serum CEA levels and tumor volume on magnetic resonance. Similarly, in our study, no significant correlation was found between the preoperative serum CEA level and MTV, TLG, and SUV $V_{\text {max }}$ values of the primary tumor. However, similar to previous reports, our findings indicate the likelihood of serum CEA levels to increase in the preoperative period $(12,18)$. Although some studies have reported that CEA positivity is not related to the tumor, tumor diameter, and TNM classification, many studies have stated that the preoperative serum CEA levels are related to the prognosis (18-22). In a retrospective study of patients with stage 2 colorectal carcinoma, Spindler et al. (19) revealed that serum CEA levels were considered to change the risk classification by contributing to the distinctive features of patients and help the management of additional treatments. In addition, Margalit et al. (20) evaluated patients with stage I and II colon cancer and stated that serum CEA level $\geq 2.35 \mathrm{ng} / \mathrm{mL}$ can be used to predict the prognosis (13). In another study, preoperative CEA levels $\geq 5$ $\mathrm{ng} / \mathrm{mL}$ were reported to be independent prognostic factor for overall survival, disease-free survival, and recurrence detection and to be associated with a high risk of mortality $(21,22)$.

Although the combined use of serum CEA levels and imaging methods increases the diagnostic sensitivity of colorectal carcinomas, many recent studies have found that postoperative serum CEA levels are clinically more valuable than its preoperative levels (23-25). Caglar et al. (10) analyzed patients with recurrent colorectal cancer and reported that all quantitative PET/CT parameters (i.e., SUV ${ }_{\max }, T L G$, and MTV) demonstrate a positive correlation with serum CEA levels (10).

\section{Study Limitations}

This study is limited by its retrospective design, small number of patients, and lack of data on comparison between benign and malign lesions in terms of CEA positivity.

\section{CONCLUSION}

In conclusion, our findings revealed no significant correlation between CEA positivity and volume-based PET/CT parameters (MTV and TLG) in the preoperative period of colon cancer. Although CEA positivity is considered useful in predicting recurrence in the postoperative period and is associated with 
tumor burden, it has low diagnostic efficacy in the preoperative period independent of tumor burden.

\section{Ethics}

Ethics Committee Approval: Permission was obtained from the Institutional Ethics Committee of the University Health Science Turkey of Gazi Yasargil Training and Research Hospital for the use and publication of patient data (protocol no: 435/2020).

Informed Consent: Before PET/CT, we routinely obtain informed consent from the patient or a relative of patient and ask whether they consent to their data being used anonymously in retrospective medical studies, and our patients in this study are patients who approve this.

Peer-review: Externally and internally peer-reviewed.

\section{Authorship Contributions}

Concept: Y.G., C.C., Design: Y.G., C.G., C.C., Data Collection or Processing: Y.G., C.G., Analysis or Interpretation: Y.G., C.G., C.C., Literature Search: C.G., C.C., Writing: Y.G., C.C.

Conflict of Interest: No conflict of interest was declared by the authors.

Financial Disclosure: The authors declared that this study received no financial support.

\section{REFERENCES}

1. Bray F, Ferlay J, Soerjomataram I, Siegel RL, Torre LA, Jemal A. Global cancer statistics 2018: GLOBOCAN estimates of incidence and mortality worldwide for 36 cancers in 185 countries. CA Cancer J Clin 2018;68:394424.

2. Gold P, Freedman SO. Specific carcinoembryonic antigens of the human digestive system. J Exp Med 1965;122:467-81.

3. Thompson JA, Grunert F, Zimmermann W. Carcinoembryonic antigen gene family: molecular biology and clinical perspectives. J Clin Lab Anal 1991:5:344-66

4. Wang JY, Lu CY, Chu KS, Ma CJ, Wu DC, Tsai HL, et al. Prognostic significance of pre- and postoperative serum carcinoembryonic antigen levels in patients with colorectal cancer. Eur Surg Res 2007;39:245-50.

5. Herbertson RA, Lee ST, Tebbutt N, Scott AM. The expanding role of PET technology in the management of patients with colorectal cancer. Ann Oncol 2007;18:1774-81.

6. Shi D, Cai G, Peng J, Li D, Li X, Xu Y, et al. The preoperative SUVmax for (18)F-FDG uptake predicts survival in patients with colorectal cancer. BMC Cancer 2015;15:991.

7. Can C. Correlation of (18F) FDG PET/CT Parameters with Haematological Parameters in Esophageal Cancers and the Effect of These Parameters on Survival. Dicle Med J 2020;47:556-67.

8. Akdeniz N, Küçüköner M, Kaplan MA, Urakçı Z, Karhan O, Yerlikaya H, et al. The Effect of Metabolic PET Parameters on Survival Outcome in Malignant Pleural Mesothelioma. Dicle Med J 2020;47:16-23.

9. Ince S, Okuyucu K, Hancerliogulları O, Alagoz E, San H, Arslan N. Clinical Significance of Fluorine-18-fluorodeoxyglucose Positron Emission Tomography/computed Tomography in the Follow-up of Colorectal
Cancer: Searching off Approaches Increasing Specificity for Detection of Recurrence. Radiol Oncol 2017;51:378-85.

10. Caglar M, Yener C, Karabulut E. Value of CT, FDG PET-CT and serum tumor markers in staging recurrent colorectal cancer. Int J Comput Assist Radiol Surg 2015;10:993-1002.

11. Dbouk HA, Tawil A, Nasr F, Kandakarjian L, Abou-Merhi R. Significance of CEA and VEGF as Diagnostic Markers of Colorectal Cancer in Lebanese Patients. Open Clin Cancer J 2007;1:1-5.

12. Siregar GA, Sibarani H. Comparison of Carcinoembryonic Antigen Levels Among Degree of Differentiation and Colorectal Cancer's Location in Medan. Open Access Maced J Med Sci 2019;7:3447-50.

13. Lee JE, Kim SW, Kim JS, Choi KY, Kang WK, Oh ST, et al. Prognostic value of 18-fluorodeoxyglucose positron emission tomography-computed tomography in resectable colorectal cancer. World J Gastroenterol 2012;18:5072-7.

14. Odalovic S, Stojiljkovic M, Sobic-Saranovic D, Pandurevic S, Brajkovic L, Milosevic I, et al. Prospective study on diagnostic and prognostic significance of postoperative FDG PET/CT in recurrent colorectal carcinoma patients: comparison with MRI and tumor markers. Neoplasma 2017;64:954-61.

15. Vallam KC, Guruchannabasavaiah B, Agrawal A, Rangarajan V, Ostwal $V$, Engineer R, et al. Carcinoembryonic antigen directed PET-CECT scanning for postoperative surveillance of colorectal cancer. Colorectal Dis 2017;19:907-11.

16. Compton CC, Fielding LP, Burgart LJ, Conley B, Cooper HS, Hamilton SR, et al. Prognostic factors in colorectal cancer. College of American Pathologists Consensus Statement 1999. Arch Pathol Lab Med 2000;124:979-94.

17. Kim YJ, Park SC, Kim DY, Moon SH, Chang HJ, Park JW, et al. No correlation between pretreatment serum CEA levels and tumor volume in locally advanced rectal cancer patients. Clin Chim Acta 2012;413:511-5.

18. Topdagi 0, Timuroglu A. Evaluation of the Relationship between Carcinoembryonic Antigen and TNM Stage in Colorectal Cancer. Eurasian J Med 2018;50:96-8.

19. Spindler BA, Bergquist JR, Thiels CA, Habermann EB, Kelley SR, Larson DW, et al. Incorporation of CEA Improves Risk Stratification in Stage II Colon Cancer. J Gastrointest Surg 2017;21:770-7.

20. Margalit O, Mamtani R, Yang YX, Reiss KA, Golan T, Halpern N, et al. Assessing the prognostic value of carcinoembryonic antigen levels in stage I and II colon cancer. Eur J Cancer 2018;94:1-5.

21. Baqar AR, Wilkins S, Staples M, Angus Lee CH, Oliva K, McMurrick P. The role of preoperative CEA in the management of colorectal cancer: A cohort study from two cancer centres. Int J Surg 2019;64:10-5.

22. Woo J, Kim J, Park I, Cho H, Gwak G, Yang KH, et al. Perioperative Serum Carcinoembryonic Antigen Ratio Is a Prognostic Indicator in Patients With Stage II Colorectal Cancer. Ann Coloproctol 2018;34:4-10.

23. Kim NH, Lee MY, Park JH, Park DI, Sohn Cl, Choi K, et al. Serum CEA and CA 19-9 Levels are Associated with the Presence and Severity of Colorectal Neoplasia. Yonsei Med J 2017;58:918-24.

24. Zhang B, Sun Z, Song M, Ma S, Tian Y, Kong Q. Ultrasound/CT combined with serum CEA/CA19.9 in the diagnosis and prognosis of rectal cancer. J BUON 2018;23:592-7.

25. Beom SH, Shin SJ, Kim CG, Kim JH, Hur H, Min BS, et al. Clinical Significance of Preoperative Serum Carcinoembryonic Antigen Within the Normal Range in Colorectal Cancer Patients Undergoing Curative Resection. Ann Surg Oncol 2020;27:2774-83. 